\title{
BMJ Open Lower levels of high-density lipoprotein cholesterol in urban Africans presenting with communicable versus non-communicable forms of heart disease: the 'Heart of Soweto' hospital registry study
}

\author{
Jasmine G Lyons, ${ }^{1,2}$ Karen Sliwa, ${ }^{3,4}$ Melinda J Carrington, ${ }^{1,4,5}$ Frederick Raal, ${ }^{6}$ \\ Sandra Pretorius, ${ }^{3}$ Friedrich Thienemann, ${ }^{3,7,8}$ Simon Stewart ${ }^{1,3,4,5}$
}

To cite: Lyons JG, Sliwa K, Carrington MJ, et al. Lower levels of high-density lipoprotein cholesterol in urban Africans presenting with communicable versus non-communicable forms of heart disease: the 'Heart of Soweto' hospital registry study. BMJ Open 2014;4: e005069. doi:10.1136/ bmjopen-2014-005069

- Prepublication history for this paper is available online. To view these files please visit the journal online (http://dx.doi.org/10.1136/ bmjopen-2014-005069).

Received 17 February 2014 Revised 22 June 2014 Accepted 24 June 2014

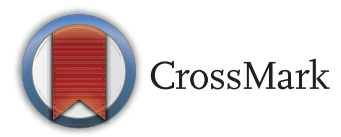

For numbered affiliations see end of article.

Correspondence to Professor Simon Stewart; simon.stewart@bakeridi. edu.au

\section{ABSTRACT}

Objectives: To investigate if urban Africans displayed lower levels of atheroprotective high-density lipoprotein cholesterol (HDLC) when presenting with communicable versus non-communicable forms of heart disease (HD) as both acute infection and chronic inflammation reduce HDLC levels.

Design: Hospital registry of 5328 de novo cases of HD over a 3-year period.

Setting: Cardiology Unit, Baragwanath Hospital in Soweto, South Africa.

Participants: A total of 1199 patients of African descent (59\% women; $57.0 \pm 13.4$ years) had fasting blood lipid levels (total cholesterol (TC), triglyceride, HDLC and low-density lipoprotein cholesterol (LDLC)) documented on admission. Serum inflammatory marker $\mathrm{C}$ reactive protein (CRP) was measured in a subset of 367 patients (31\% of cases).

Main outcome measures: Lipid profiles were compared according to prespecified classification of non-communicable (eg, hypertensive HD) versus communicable (eg, rheumatic HD) HD. Low HDLC was defined as $<1.0 \mathrm{mmol} / \mathrm{L}$ for men and $<1.2 \mathrm{mmo} / \mathrm{L}$ for women, according to applicable South African Clinical Guidelines.

Results: Overall 694 (58\%) of those presenting with HD had low HDLC levels; 344 of $678(51 \%)$ and 350 of $521(67 \%)$ for non-communicable and communicable, respectively $(p<0.001)$. Comparatively, overall prevalence of high TC was $32 \%$ and high LDLC was $37 \%$. On an adjusted basis, those with noncommunicable HD were more likely to record a low HDLC relative to non-communicable presentations (odds ratio (OR) $1.91,95 \% \mathrm{Cl} 1.42$ to $2.57 ; \mathrm{p}<0.001$ ). There was a strong relationship between low HDLC and higher levels of CRP, but only in women.

Conclusions: Despite largely favourable lipid profiles, there are clear differences according to aetiology of underlying $\mathrm{HD}$ in urban Africans, with younger patients with communicable HD having particularly low levels of HDLC. Appropriate prospective evidence is needed to

\section{Strengths and limitations of this study}

- We report a high prevalence of low high-density lipoprotein cholesterol (HDLC) in de novo presentations of both communicable heart disease and non-communicable heart disease, with a greater prevalence in those with communicable heart disease.

- The study cohort is clinically very well defined; however the lipid data were obtained according to clinical presentation, which may impose systematic bias to the results.

- This hospital registry study has provided preliminary data that would support prospective investigation of longer-term dyslipidaemia patterns and their impact on heart disease incidence, both in South Africa and in other low-and-middleincome countries where the epidemiologic transition is currently underway.

determine if persistent low levels of HDLC expose patients to increased, long-term risk of atherosclerotic forms of HD. The women-only inverse association between HDLC and CRP warrants further investigation.

\section{INTRODUCTION}

Heart diseases (HDs) with infectious aetiology have long been the principal forms of cardiovascular disease (CVD) in sub-Saharan Africa. However, epidemiological transition has seen increased prevalence of noncommunicable forms of HD in these populations. ${ }^{1}$ This phenomenon is largely driven by complex, population-wide changes in demographic, social and economic status, with associated changes in lifestyle habits. ${ }^{2-4}$ Indicative of the tension between 'old' and 
'new' forms of $\mathrm{HD}$, the incidence of communicable heart disease $(\mathrm{CD})$ is sustained by the devastating epidemics of HIV/AIDS, tuberculosis (TB), rheumatic heart disease (RHD) and parasitic infections with cardiac involvement, ${ }^{5-7}$ while in parallel, the prevalence of risk factors for non-communicable heart disease (NCD) increases. ${ }^{8}$

Low serum levels of high-density lipoprotein cholesterol (HDLC) are consistently and independently associated with increased risk of atherosclerotic forms of CVD. ${ }^{9}{ }^{10}$ However, it remains uncertain whether low HDLC is causal or just a cardiovascular risk marker. ${ }^{11}$ If we are to extrapolate from studies in Western and Asian populations, ${ }^{12-14}$ isolated low HDLC is associated with increased risk for CVD in the long-term. There are several causes of low HDLC levels, including overweight, obesity, tobacco smoking and insulin resistance/type 2 diabetes mellitus, indicative of the important role lifestyle factors have in mediating HDLC levels. ${ }^{9}$ Additionally, low HDLC is a striking consequence of abnormal lipid metabolism in infection and inflammation. ${ }^{15}$ Although it has been shown that those of African descent largely show a favourable lipid profile characterised by high HDLC levels,${ }^{16}$ it is unlikely that they can remain atheroprotective during an infected state. ${ }^{15}$ Indeed, in this setting, it is probable that increasing prevalence of modifiable/lifestyle risk factors contribute to a more advanced presentation in those with $\mathrm{CD} .{ }^{8}$ We have previously reported that in the geographically compact townships that comprise Soweto in South Africa, 'old' and 'new' forms of HD are simultaneously present. ${ }^{8}$ While this tension exists, we have a unique opportunity to explore lipid profiles in patients presenting with non-communicable versus communicable forms of HD.

\section{STUDY HYPOTHESES}

Having shown important ethnic differences in the lipid profiles of patients of African descent presenting with HD in the urban African enclave of Soweto, ${ }^{16}$ we hypothesised that independent of age and sex, urban Africans presenting with $\mathrm{CD}$ will demonstrate patterns of dyslipidaemia associated with infection/inflammation, particularly suboptimal levels of HDLC, versus NCD.

\section{METHODS}

\section{Study setting and design}

As described in detail previously ${ }^{8} 17$ the 3500 bed Chris Hani Baragwanath Hospital (case load of $>125000$ inpatients per annum) services the tertiary care needs of Soweto (population of 1.1 million) and surrounding communities. All suspected cardiac presentations are referred to the hospital's Cardiology Unit for advanced diagnostic testing and gold-standard treatments. A prospective clinical registry of all de novo presentations of the same was established in 2006 as part of the Heart of Soweto Study and represents sub-Saharan Africa's largest and most detailed study of advanced forms of HD to date. ${ }^{17}$

\section{Participants}

The 'Heart of Soweto' cohort of de novo case presentations comprised of 5328 patients. Of these, 2185 patients $(40 \%)$ had a documented fasting lipid profile (serum total cholesterol (TC) level, triglyceride (TG), HDLC and calculated low-density lipoprotein cholesterol $\left(\right.$ LDLC) ${ }^{18}$ undertaken at Baragwanath Hospital on-site pathology). None of the patients were on lipid lowering agents at the time of presentation, as this medication can only be prescribed at the tertiary institution. However, some of the patients had been placed on antihypertensive medication prior to their first assessment at the Cardiac Clinic at Baragwanath Hospital. Moreover, only a small number of patients (39 cases) had been prescribed antiretroviral therapy (ART) on presentation. All patients provided informed consent.

\section{Study data}

A complete list of study data captured by the registry, comprising basic sociodemographic (including selfreported ethnicity, years of education and determining if the patient was born in Soweto) and advanced clinical profiling, has been described previously. ${ }^{8} 17$ The registry captured all advanced clinical investigative procedures (eg, coronary angiography, which was undertaken in all people diagnosed with coronary artery disease (CAD)). Echocardiography (performed on all patients) criteria used in the study have been described in detail previously. ${ }^{817}$

\section{Case classifications}

Adjudication and classification of communicable and non-communicable presentations of HD in this cohort have been previously described. ${ }^{8}$ After exclusion of those with uncomplicated hypertension (ie, without evidence of cardiac dysfunction, $n=380$ ) or other nonmodifiable aetiologies (eg, congenital disorders), 1199 patients of African descent (22\% of the total 'Heart of Soweto' cohort) were included in this analysis. Contributory diagnoses for NCD were predominantly hypertensive heart failure (HT-HF) and CAD (without HIV). CD was predominantly classified as HIV-dilated cardiomyopathy (HIV-DCMO), HIV-pulmonary hypertension, TB pericardial disease and pericarditis due to other infections.

\section{Risk factor definition}

Optimum lipid levels and treatment goals with established CVD were defined according to international guidelines ${ }^{9}$ adopted by the Lipid and Atherosclerosis Society of South Africa and the South African Heart Association-high TC: $>4.5 \mathrm{mmol} / \mathrm{L}$, high TGs: $>1.7 \mathrm{mmol} / \mathrm{L}$, high LDLC: $>2.5 \mathrm{mmol} / \mathrm{L}$ and low HDLC: $<1.0$ for men and $<1.2 \mathrm{mmol} / \mathrm{L}$ for women. ${ }^{19}$ Other risk factors were measured on a clinical basis, as previously described. ${ }^{17}$ Anthropometric measurements were available for calculation of body mass index (BMI, $\mathrm{kg} / \mathrm{m}^{2}$ ) in $854(71 \%)$ cases, the low reporting rate restricted to 
ambulatory patients. Obesity was defined as BMI $\geq 30 \mathrm{~kg} / \mathrm{m}^{2}$. Serum C reactive protein (CRP) was measured in a subset of 367 patients (31\% of all cases) if clinically indicated (eg, suspected infection). Patients were stratified into clinically relevant CRP categories, ${ }^{20}$ as defined by Dhingra et $a l^{21}$ Patients with a CRP of $1 \mathrm{mg} / \mathrm{L}(\mathrm{n}=19)$ were used as reference group and compared with medium (1.1-3.0 mg/L, n=26), high (3.1$10.0 \mathrm{mg} / \mathrm{L}, \mathrm{n}=83)$ and very high $(>10.0 \mathrm{mg} / \mathrm{L}, \mathrm{n}=239)$ CRP categories.

\section{Statistical analyses}

Normally distributed continuous data are presented as the mean $\pm \mathrm{SD}$ and non-Gaussian distributed variables as the median (IQR). Categorical data are presented as sample number and percentages. For group comparisons, we initially used $\chi^{2}$ analysis with calculation of odds ratios (ORs) with 95\% CIs presented were appropriate for discrete variables, and independent $t$ tests for normally distributed continuous variables and Mann-Whitney $\mathrm{U}$ test for non-parametric continuous variables. Multiple logistic regression analyses (entry model) were used to derive age and sex adjusted ORs (and BMI in some analyses, as described) for the risk of presenting with clinically relevant variables (primarily dyslipidaemia profiles), according to CD HD relative to NCD diagnosis. Significance was accepted at the twosided level of $\mathrm{p}<0.05$.

\section{RESULTS}

\section{Clinical and demographic profile}

Table 1 shows the sociodemographic and clinical profile of this cohort according to cardiac aetiology. Those presenting with NCD ( $\mathrm{n}=678,56.5 \%$ of cohort) were older and had higher BMI and mean systolic blood pressure (SBP) and diastolic blood pressure (DBP) than those with $\mathrm{CD} \quad(\mathrm{n}=521,43.5 \%$; all comparisons $\mathrm{p}<0.001)$. Overall, $76(6 \%)$ were confirmed HIV positive: 15 (2\%) and $61(12 \%)$ patients were confirmed HIV positive in $\mathrm{NCD}$ and CD groups, respectively $(\mathrm{p}<0.001)$. Apart from higher BMIs in women $\left(30.6 \pm 6.9\right.$ vs $26.7 \pm 5.5 \mathrm{~kg} / \mathrm{m}^{2}$ in men, $\mathrm{p}<0.001)$ there were no significant differences between sexes with respect to other clinical parameters. To this, the prevalence of obesity in women was $50 \%$ as compared with men $(26 \%), \mathrm{p}<0.001$.

\section{Aetiology of HD (primary diagnosis)}

Overall, the most prevalent primary diagnoses were HT-HF $(n=461,38 \%)$, DCMO $(n=178,15 \%)$ and CAD $(\mathrm{n}=157,12 \%)$. In those classified as NCD, HT-HF was the main primary diagnosis $(n=461,68 \%)$, along with CAD (without concurrent HIV infection; $\mathrm{n}=157,23 \%$ ). DCMO $(\mathrm{n}=178,34 \%)$, right $\mathrm{HF}(\mathrm{n}=92,18 \%)$ and right HD $(n=63,12 \%)$ and other forms of primary valve disease $(n=71,14 \%)$, were the most common diagnoses in those classified with communicable forms of HD.
Lipid profiles

Those with CD have significantly lower TC, LDLC and HDLC compared with patients with NCD (table 1 and figure $1, \mathrm{p}<0.001$ for all comparisons). Overall, women had significantly higher TC $(4.2 \pm 1.3$ vs $3.8 \pm 1.2 \mathrm{mmol} / \mathrm{L}$, $\mathrm{p}<0.001)$, LDLC $(2.4 \pm 1.0$ vs $2.2 \pm 1.0 \mathrm{mmol} / \mathrm{L}, \mathrm{p}<0.01)$ and HDLC compared with men $(1.2 \pm 0.5$ vs 1.0 $\pm 0.5 \mathrm{mmol} / \mathrm{L}, \mathrm{p}<0.001)$. This gender difference did not extend to TGs $(1.1(0.4-1.8)$ vs $1.1(0.4-1.8) \mathrm{mmol} / \mathrm{L}$, $\mathrm{p}=0.7)$ nor TC: $\mathrm{HDLC}$ ratio $(4.2 \pm 3.1$ vs $4.3 \pm 2.7 \mathrm{mmol} / \mathrm{L}$, $\mathrm{p}=0.6$ ). Lipid ratios were calculated and compared (table 1). There were no significant differences between aetiology groups for either TC:HDL or TG:HDL groups. However LDL:HDL ratios were significantly higher in the communicable group.

Levels of TC (figure 1A), HDLC (figure 1B) and LDLC (figure 1C) were significantly higher in women with NCD (figure 1). However, in those diagnosed with $\mathrm{CD}$, small, but significant, differences were observed only for TC and HDLC, not LDL (figure 1). Overall, prevalence of dyslipidaemia varied from $18 \%$ of patients with high TGs to $58 \%$ with low HDLC (table 1 and figure 2). Consistent with the decrease observed with the actual levels, prevalence of high TC and high LDLC was increased in those with NCD aetiologies while low HDLC levels prevalence was higher in those with CD (table 1 and figure 2). There were no patients with TG levels $>4.5 \mathrm{mmol} / \mathrm{L}$ (range $0.1-3.8 \mathrm{mmol} / \mathrm{L}$ ) which makes use of the Friedewald equation suitable for this cohort. $^{18}$

Table 2 shows independent associations between relevant socioeconomic, demographic and clinical variables and $\mathrm{CD}$ aetiology, relative to those presenting with NCD. The effect of HD aetiology on low HDLC dyslipidaemia was strong and consistent: adjusting for age, sex and BMI of patients, those with forms of CD were significantly more likely to record a low HDLC relative to those presenting with $\mathrm{NCD}$ (table 2, $\mathrm{p}<0.001$ ) and less likely to record high TC and LDLC (table 2). Patients with $\mathrm{CD}$ were less likely to record high TG levels (OR $0.65,95 \%$ CI 0.51 to $0.84, \mathrm{p}<0.05$ ) compared to those with NCD.

\section{CRP subset analysis}

Overall, there was no significant difference in CRP levels between aetiology groups (table 1). The proportion of confirmed HIV cases in this CRP subset analysis was 7\% $(n=27)$. Of those, 23 were in the very high-risk category. There was also no association between CRP-derived risk categories and high TC, LDLC or TGs (data not shown). However, the risk of having low HDLC increased with increasing CRP levels. In age and sex-adjusted analyses, those with medium risk (OR 2.73, 95\% CI 0.68 to 10.89 , $\mathrm{p}=0.16$ ), high risk (OR 4.98, 95\% CI 1.46 to 17.00 , $\mathrm{p}=0.01$ ) and very high risk (OR 6.37, 95\% CI 1.97 to 20.57, $\mathrm{p}<0.01$ ) CRP levels were significantly more likely to record a low HDLC relative to those in the low risk CRP group. In addition, when stratified by sex, a 
Table 1 Clinical and demographic profile according to heart disease aetiology.

\begin{tabular}{|c|c|c|c|c|}
\hline & $\begin{array}{l}\text { All cases } \\
n=1199\end{array}$ & $\begin{array}{l}\text { Non-communicable } \\
n=678(57 \%)\end{array}$ & $\begin{array}{l}\text { Communicable } \\
n=521(43 \%)\end{array}$ & p Value \\
\hline \multicolumn{5}{|l|}{ Demographic profile } \\
\hline Mean age (years) & $58.3 \pm 14.0$ & $60.1 \pm 13.1$ & $55.9 \pm 14.9$ & $<0.001$ \\
\hline Women & $701(59 \%)$ & $403(59 \%)$ & $298(57 \%)$ & 0.44 \\
\hline$<6$ years formal education & $582(49 \%)$ & $335(49 \%)$ & $247(47 \%)$ & 0.52 \\
\hline Soweto origin & $562(47 \%)$ & $327(48 \%)$ & $235(45 \%)$ & 0.29 \\
\hline \multicolumn{5}{|l|}{ Clinical presentation } \\
\hline Total cholesterol (mmol/L) & $4.0 \pm 1.4$ & $4.3 \pm 1.3$ & $3.7 \pm 1.2$ & $<0.001$ \\
\hline $\mathrm{HDLC}(\mathrm{mmol} / \mathrm{L})$ & $1.1 \pm 0.5$ & $1.2 \pm 0.5$ & $1.0 \pm 0.5$ & $<0.001$ \\
\hline Median triglycerides $(\mathrm{mmol} / \mathrm{L})^{*}$ & $1.1(0.8,1.5)$ & $1.1(0.8,1.6)$ & $1.0(0.7,1.3)$ & $<0.001$ \\
\hline $\operatorname{LDLC}(\mathrm{mmol} / \mathrm{L})$ & $2.4 \pm 1.0$ & $2.5 \pm 1.0$ & $2.2 \pm 0.9$ & $<0.001$ \\
\hline TC : HDLC ratio & $4.3 \pm 3.0$ & $4.2 \pm 3.1$ & $4.4 \pm 2.7$ & 0.27 \\
\hline LDL : HDLC ratio & $2.5 \pm 1.1$ & $2.5 \pm 1.0$ & $2.7 \pm 1.1$ & 0.36 \\
\hline TG : HDLC ratio* & $1.1(0.7,1.7)$ & $1.0(0.7,1.7)$ & $1.1(0.7,1.8)$ & 0.12 \\
\hline Median serum CRP (mg/L)* & $19(7.0,45.0)$ & $16.8(6.6,41.5)$ & $20.5(7.8,55.9)$ & 0.25 \\
\hline Systolic BP (mm Hg) & $135 \pm 29$ & $143 \pm 29$ & $126 \pm 26$ & $<0.001$ \\
\hline Diastolic BP (mm Hg) & $78 \pm 16$ & $80 \pm 16$ & $74 \pm 16$ & $<0.001$ \\
\hline $\mathrm{BMI}\left(\mathrm{kg} / \mathrm{m}^{2}\right)$ & $29.0 \pm 6.7$ & $30.3 \pm 6.7$ & $27.2 \pm 6.2$ & $<0.001$ \\
\hline \multicolumn{5}{|l|}{ Prevalence of dyslipidaemia (n, \%) } \\
\hline High total cholesterol (>5 mmol/L) & $378(32 \%)$ & $266(39 \%)$ & $112(22 \%)$ & $<0.001$ \\
\hline Low HDLC ( $<1$ in men and $<1.2 \mathrm{mmol} / \mathrm{L}$ in women) & $694(58 \%)$ & $344(51 \%)$ & $350(67 \%)$ & $<0.001$ \\
\hline High LDLC (>2.5 mmol/L) & $446(37 \%)$ & $291(43 \%)$ & 155 (30\%) & $<0.001$ \\
\hline High triglycerides (>1.7 mmol/L) & $215(18 \%)$ & $143(21 \%)$ & $72(14 \%)$ & 0.001 \\
\hline \multicolumn{5}{|l|}{ Prevalence of other risk factors $(n, \%)$} \\
\hline Obese $\left(\mathrm{BMI}>30 \mathrm{~kg} / \mathrm{m}^{2}\right)$ & $344(40 \%)$ & $237(48 \%)$ & $107(30 \%)$ & $<0.001$ \\
\hline Type 2 diabetes & $98(8 \%)$ & $71(11 \%)$ & $27(5 \%)$ & $<0.001$ \\
\hline Past or current smoker & $566(47 \%)$ & $321(47 \%)$ & $245(47 \%)$ & 0.95 \\
\hline Family history of CVD & $466(39 \%)$ & $286(42 \%)$ & $180(35 \%)$ & 0.01 \\
\hline Confirmed HIV-positive cases & $76(6 \%)$ & $15(2 \%)$ & $61(12 \%)$ & $<0.001$ \\
\hline
\end{tabular}

*Median (IQR) values presented, differences tested by Mann-Whitney $U$ test.

$\mathrm{BMI}$, body mass index (available in 854 cases); BP, blood pressure; CRP, C reactive protein (available in 367 cases); CVD, cardiovascular disease; HDLC, high-density lipoprotein cholesterol; LDLC, low-density lipoprotein cholesterol; TG, triglycerides.

strong, positive association remained in women but was no longer apparent in men (figure 3). In women, the pattern was significant across all CRP risk categories: compared to low risk, those with medium risk (OR 12.1, $95 \%$ CI 1.21 to $120, \mathrm{p}=0.03)$, high risk (OR $14.4,95 \% \mathrm{CI}$ 1.64 to $126, \mathrm{p}=0.02$ ) and very high risk (OR 23.5, 95\% CI 2.81 to $197, \mathrm{p}=0.004)$ CRP levels were all more likely to record a low HDLC. Moreover, the association was not weakened by addition of BMI into the model (BMI and CRP measurements available in only 230 cases) in overall and women-only $(\mathrm{n}=133)$ models: those with medium risk (OR 20.5, 95\% CI 1.72 to $246, \mathrm{p}=0.02$ ), high risk (OR 10.6, 95\% CI 1.14 to 98.8, $\mathrm{p}=0.04$ ) and very high risk (OR 21.0, 95\% CI 2.38 to $185, \mathrm{p}<0.01$ ) CRP levels were all more likely to record a low HDLC.

\section{DISCUSSION}

We report significant decreases in lipid levels (TC, HDLC and LDLC) and age and BMI according to noncommunicable and communicable manifestations of de novo HD in urban Africans, patterns that were observed in both sexes. The high prevalence of low HDLC in more than half of all cases, but much higher in those with $\mathrm{CD}$, is most striking. In addition, it appears that gender is an effect modifier in the relationship between CRP and low HDLC in this cohort, but, importantly, the relationship remains even after adjustment for the significant confounder of adiposity.

While traditionally uncommon, ${ }^{22}$ dyslipidaemia, in particular low HDLC, is becoming more prevalent in sub-Saharan Africa. ${ }^{23}$ The low lipid levels present in the majority of cases with CD reflect the dramatic changes to lipid metabolism observed in infection and is therefore, anticipated. We acknowledge that atherogenic LDLC is also low in this setting and that low HDLC may not be indicative of particularly increased disease risk, at least in the short-term. However, we still deem this as highly clinically relevant given that even isolated low HDLC is associated with a higher risk of atherosclerotic forms of HD, a finding that has been seen in diverse populations. ${ }^{12-14}$ Interestingly TG levels were not significantly increased in those with communicable forms of HD, despite evidence that it can increase as part of the infectious/inflammatory metabolic milieu. ${ }^{15}$ Additionally, we speculate that the higher lipid levels in women may be the result of much higher rates of obesity ( $50 \%$ compared with $26 \%$ in men) as the driver 

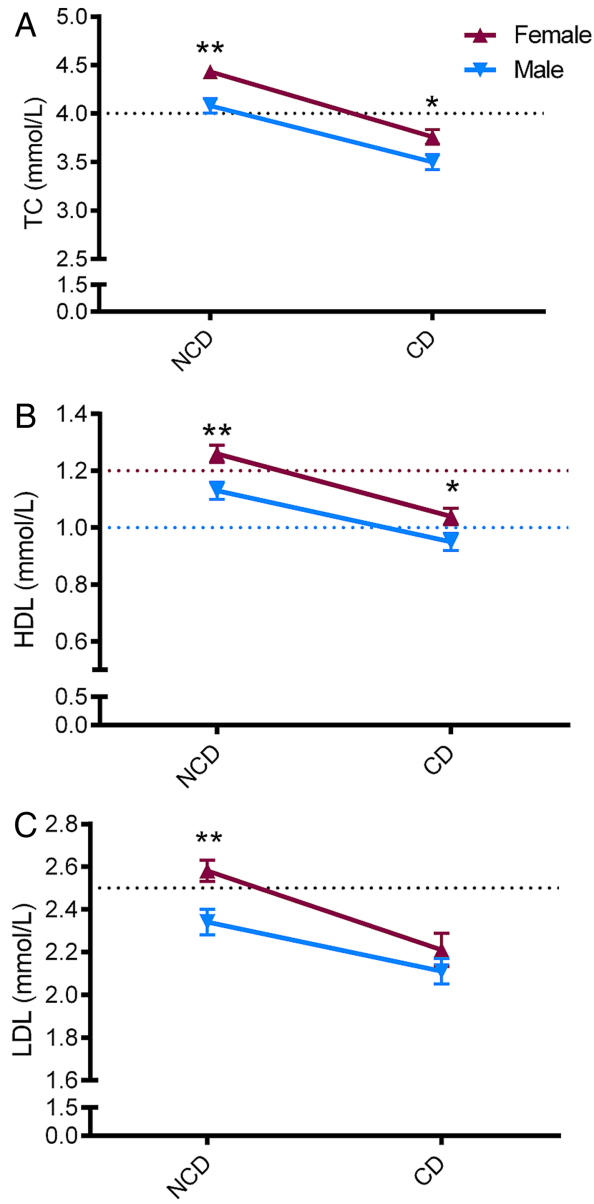

Figure 1 Sex specific lipid profiles according to heart disease aetiology. Lipid values are shown as mean \pm SE. $p$ Values indicate between-sex comparisons per aetiology group (t test), ${ }^{* *} p<0.01 ;{ }^{*} p<0.05$. NCD, non-communicable heart disease; $C D$, communicable heart disease; TC, total cholesterol; HDL, high-density lipoprotein; LDL, low-density lipoprotein. Y-axis dotted lines show thresholds for high TC and LDL or low HDL (sex specific values).

of elevated TC, which has been suggested by authors of a worldwide systematic analysis on high TC. ${ }^{25}$

Our interest in this phenomenon predominantly relates to the longer-term effects of low HDLC, especially when observed together with the amplified vascular risk associated with chronic infection. ${ }^{15}$ In Africa, where acute coronary syndromes are seen in a relatively young population, ${ }^{26}$ we predict the very high rates of myriads of communicable diseases ${ }^{27} 28$ will result in more complex cases, with potentially poorer outcomes in the long-term, given the critical role of HDLC in both innate and adaptive immunity. ${ }^{15}$ While many infectious diseases (bacterial and viral) have contributed to the underlying pathology of HD reported here ${ }^{5}$ dyslipidaemia associated with HIV infection has been particularly well studied. HIV-related low HDLC is likely a consequence of both the viral infection and an adverse effect of some antiretroviral treatment regimens, ${ }^{28-31}$ however only 39 patients of the $76(51 \%)$ confirmed HIV positive

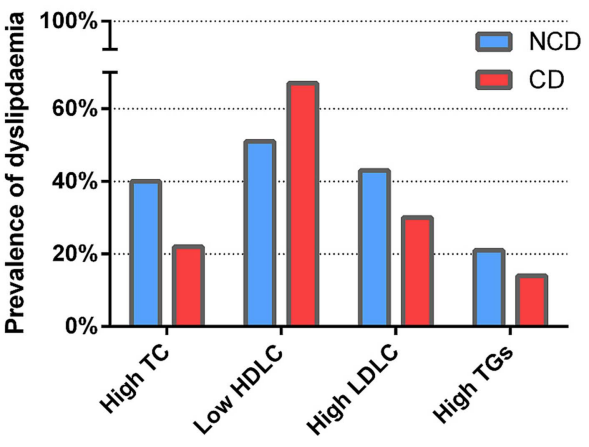

Figure 2 Prevalence of low high-density lipoprotein cholesterol according to heart disease aetiology. NCD, non-communicable heart disease; $C D$, communicable heart disease. High total cholesterol (TC) $>4.5 \mathrm{mmol} / \mathrm{L}$; Low high-density lipoprotein cholesterol (HDLC) $(<1.0 \mathrm{mmol} / \mathrm{L}$ in men, $<1.2 \mathrm{mmol} / \mathrm{L}$ in women). High low-density lipoprotein cholesterol (LDLC) >2.5 mmol/L; High triglycerides (TGs) $>1.7 \mathrm{mmol} / \mathrm{L}$.

were on ART at the time of presentation, representing $3 \%$ of the entire subset sample, which possibly dilutes this effect.

Our CRP subset analysis found associations between low HDLC and the pro-inflammatory marker CRP in patients with newly diagnosed HD in a sex disparate manner, with a much stronger positive association in women. Median CRP levels were also very high across all categories of HD aetiology, and are much higher than previous reports in early analyses of large cohorts ${ }^{20}$ as well as South African studies, ${ }^{32}$ but reflect the clinical requirements at presentation. These high levels may also be the result of 'multimorbidity' observed in the cohort, given the prevalence of infectious disease (such as HIV/ AIDS) as well as other lifestyle factors that can also influence CRP levels; ${ }^{33}$ all of which may have contributed to

Table 2 Independent correlates of communicable heart disease, relative to non-communicable heart disease.

\begin{tabular}{lcc}
\hline & \multicolumn{2}{c}{ Communicable disease } \\
\cline { 2 - 3 } & OR & $\mathbf{9 5 \%}$ Cl \\
\hline Women & 0.91 & 0.72 to 1.15 \\
Age & 0.98 & 0.97 to $0.99^{\star *}$ \\
Obesity & 0.50 & 0.37 to $0.68^{\star \star *}$ \\
<6 years formal education & 1.11 & 0.88 to 1.42 \\
Soweto origin & 0.98 & 0.77 to 1.24 \\
Body mass index adjusted analysis & \\
$\quad$ High TC & 0.52 & 0.37 to $0.71^{\star \star *}$ \\
High LDLC & 0.56 & 0.41 to $0.76^{\star \star *}$ \\
Low HDLC & 1.91 & 1.42 to $2.57^{\star \star *}$ \\
High TG & 0.65 & 0.51 to $0.84^{*}$
\end{tabular}

Obesity $\mathrm{BMI}>30 \mathrm{~kg} / \mathrm{m}^{2}$; High total cholesterol $(\mathrm{TC})>4.5 \mathrm{mmol} / \mathrm{L}$; High low-density lipoprotein (LDLC) $>2.5 \mathrm{mmol} / \mathrm{L}$; Low high-density lipoprotein (HDLC) $(<1.0 \mathrm{mmol} / \mathrm{L}$ in men, $<1.2 \mathrm{mmol} / \mathrm{L}$ in women). Age and sex-adjusted analysis: ${ }^{*} p<0.05 ;{ }^{* *} p<0.01$; ${ }^{* * *} p<0.001$. 


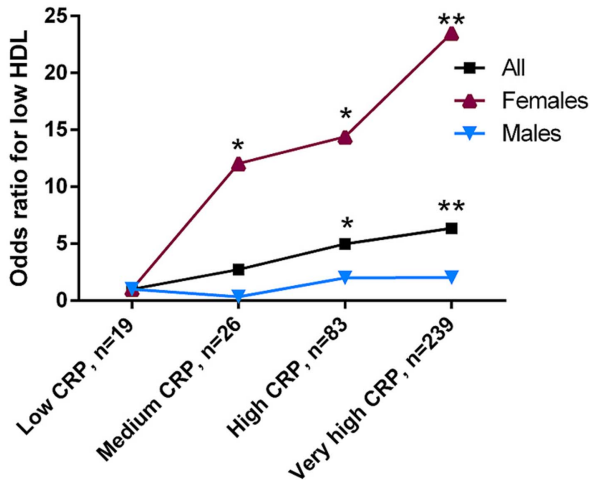

Figure 3 Risk of low high-density lipoprotein cholesterol according to $C$ reactive protein risk group, relative to low-risk $C$ reactive protein group $(n=367)$. Age-adjusted analysis. $\mathrm{CRP}=\mathrm{C}$ reactive protein. ${ }^{\star *} \mathrm{p}<0.01 ;{ }^{*} \mathrm{p}<0.05$ relative to low CRP group. For Cls, please refer to the Results section.

the high levels observed. Inflammatory stress may be having a more adverse effect on HDLC in women compared to men as a result of many causes. The prognostic value of stratifying CVD risk, even at very high CRP $(>10 \mathrm{mg} / \mathrm{L})$ levels, has been demonstrated in a very large cohort of women, ${ }^{20}$ and there are reports of elevated CRP in women of African descent. ${ }^{34}$ We also report women as having a significantly higher BMI; obesity itself can induce a low-grade inflammatory response, however the association between low HDLC and CRP in women remained even after adjusting for BMI. While we have assumed that the exaggerated drop in HDLC in women with acute forms of $\mathrm{CD}$ is a consequence rather than a cause of infection, treatment of atherogenic dyslipidaemia and inflammatory markers in women are of particular clinical relevance in a setting where obesity and its antecedent behaviours are increasing.

These results underscore the need to consider multifactorial CVD risk burden that recognises that co-occurrence of infectious and non-communicable disease produces significant and complex health disparities. Certainly, the clinical strategies to protect the heart and vessels in acute infection differ from those required in chronic infection and it is unlikely that lipid measurements will form a cornerstone of treatment in such cases. However, it is important to recognise the benefits of early detection and treatment of dyslipidaemia in order to mitigate any double effect of infectious and 'lifestyle' HD risk factors in the longer term. While the epidemiological evidence is clear, the precise mechanism by which HDLC decreases atherosclerotic CVD risk remains unclear; ${ }^{11}$ indeed, efforts to develop pharmacological modalities to specifically increase HDLC levels to reduce cardiovascular risk, continues to be problematic ${ }^{10}$ and we acknowledge that addressing the low HDLC observed in this cohort, in isolation, without commensurate improvements in HDLC functionality, will prove a difficult task. This does not, however, preclude the use of other therapeutic interventions that address the greater, more complex risk presentation of cases that fall in the "crossover' between communicable and non-communicable diseases. For example, the polypill, which includes lipid-lowering medications, has been proposed as a viable treatment option in secondary prevention, given its relative ease of use and efficacy in low-income settings. ${ }^{35}$ More so, evidence that statins also exert immunomodulatory effects, along with suggestions they may prove useful in the treatment and prevention of infections, ${ }^{36}$ indicate they may have important, multifaceted clinical implications in populations such as Soweto, especially given the substantial dyslipidaemic risk associated with highly prevalent HIV infection and ART. Attempts to address prevention, management, cure and control of noncommunicable and communicable forms of HD as entirely separate entities are likely to prove insufficient. This holds true on a per-patient basis as well as for any population-wide, public health approach.

There are a number of limitations that require consideration. Clinical data (other than routine echocardiography and 12-lead ECG) were obtained according to presentation. This study was not specifically designed to comprehensively delineate between specific forms of HD (resulting in variable clinical data) although this is part of clinical investigation at Baragwanath Hospital; although it should be noted HIV status is not routinely determined. The arbitrary selection of disease states into the communicable versus non-communicable groups (eg, primary valve disease) may be questioned; hence our further delineation of clearly identifiable cases of acute inflammation/infection at the point of admission. However, we would emphasise that classification was prospectively applied, the groupings are consistent with our previous reports that describe in detail the rigorous clinical criteria employed in profiling the 'Heart of Soweto' cohort, and expected gradients in lipid levels were subsequently found. Systematic bias needs to be carefully considered before attributing broad patterns in lipid profiles, as those with suspected atherosclerotic disease were more likely to have had lipid levels measured, reflecting the low number of those presenting an acute infectious form of HD (eg, patients with pericarditis). Adiposity, a major confounder of dyslipidaemia and HD, was recorded in $71 \%$ of the cohort. However, its inclusion in the regression analyses did not alter the significance of the associations. Central obesity measurements (eg, waist-to-hip ratio) may have offered greater delineation of CVD risk but data were not available. CRP was measured in just under one third of cases and related data require careful interpretation. Finally, owing to the cross-sectional design of this study we were not able to investigate the possible effect of the magnitude and timing of the contributing infection on lipid levels, beyond the data collected at admission. Given the transient, dynamic processes of lipid metabolism over the course of acute and chronic diseases, only longitudinal studies of lipid levels and subsequent outcomes can fully elucidate the clinical importance of our findings. 


\section{CONCLUSIONS}

We have shown that despite largely favourable lipid profiles, there are clear differences according to underlying aetiology of HD in urban Africans however, overall low HDLC was the most prevalent metabolic abnormality observed in this cohort. Younger Africans with CD have particularly low levels of HDLC that, if maintained in the longer term, may leave them at increased risk of atherosclerotic disease. This is physiologically plausible in chronic infection; however low HDL at hospital admission could also simply reflect similarly low levels of TC/ LDL and may not be indicative of increased long-term GVD risk. That uncertainty can only be resolved by wellpowered studies with adequate follow-up, to provide sufficient evidence to address current gaps in evidence and, ultimately, guide clinical practice. Nevertheless, if proven, targeted prevention programmes that identify and actively manage individuals with a history of CD (particularly an active case) and with low levels of HDLC may be indicated. The alternative is an increasing burden of non-communicable forms of HD in urban African communities that is supplemented (in origin and confluence) by historical cases of communicable disease that have adversely affected protective HDLC levels ( particularly in women).

\author{
Author affiliations \\ ${ }^{1}$ Baker IDI Heart and Diabetes Institute, Melbourne, Victoria, Australia \\ ${ }^{2}$ Division of Health Sciences, University of South Australia, Adelaide, Australia \\ ${ }^{3}$ Hatter Institute for Cardiovascular Research in Africa, Cape Heart Group and \\ IIDMM, University of Cape Town, Cape Town, South Africa \\ ${ }^{4}$ Soweto Cardiovascular Research Unit, University of the Witwatersrand, \\ Johannesburg, South Africa \\ ${ }^{5}$ Department of Epidemiology and Preventative Medicine, Monash University, \\ Melbourne, Australia \\ ${ }^{6}$ Carbohydrate and Lipid Metabolism Research Unit, University of the \\ Witwatersrand, Johannesburg, South Africa \\ ${ }^{7}$ Institute of Infectious Diseases and Molecular Medicine, University of Cape \\ Town, Cape Town, South Africa \\ ${ }^{8}$ Infectious Diseases Referral Clinic, GF Jooste Hospital, Manenberg, \\ South Africa
}

Acknowledgements The authors thank all the doctors, nurses and patients who participated in the registry; and Elisabeth Tshele, Bridget Phooko, Maureen Kubheka and Phutuma Mathusi who contributed to the meticulous collection and management of clinical data.

Contributors KS, MJC and SS participated in the original design of the study and supervised the collection of data. JGL prepared the first draft of the manuscript, with edits and revisions provided by all authors. FR and FT revised manuscript critically for important intellectual content. All authors had full access to all the data and read and approved the final version of the manuscript. All authors had final responsibility for the decision to submit the manuscript for publication.

Funding The 'Heart of Soweto Study' registry was supported by the University of the Witwatersrand and unconditional research grants from Adcock-Ingram, the Medtronic Foundation USA, Servier, Bayer-Schering and BHP Billiton. All authors had financial support from independent funding bodies, including University of the Witwatersrand and unconditional research grants from Adcock-Ingram, the Medtronic Foundation USA, Servier, BayerSchering and BHP Billiton, for the submitted work. SS, MJC and JGL are supported by the National Health and Medical Research Council of Australia [Programme Grants 320860 and 631947 and Postgraduate scholarship
586739]. JGL is supported by the National Heart Foundation of Australia and Cardiac Society of Australia and New Zealand. Baker IDI is supported by the Victorian Government's Operational Infrastructure Support Programme. KS and SL are supported by the MRC South Africa and the University of Cape Town.

Competing interests None.

\section{Patient consent Obtained.}

Ethics approval The study was approved by the University of the Witwatersrand Ethical Committee and conforms to the principles outlined in the Declaration of Helsinki.

Provenance and peer review Not commissioned; externally peer reviewed.

Data sharing statement Study data will be available on request from the corresponding author.

Open Access This is an Open Access article distributed in accordance with the Creative Commons Attribution Non Commercial (CC BY-NC 3.0) license, which permits others to distribute, remix, adapt, build upon this work noncommercially, and license their derivative works on different terms, provided the original work is properly cited and the use is non-commercial. See: http:// creativecommons.org/licenses/by-nc/3.0/

\section{REFERENCES}

1. Yusuf S, Reddy S, Ounpuu S, et al. Global burden of cardiovascular diseases: part II: variations in cardiovascular disease by specific ethnic groups and geographic regions and prevention strategies. Circulation 2001;104:2855-64.

2. Vorster $\mathrm{HH}$. The emergence of cardiovascular disease during urbanisation of Africans. Public Health Nutr 2002;5:239-43.

3. Mensah GA. Descriptive epidemiology of cardiovascular risk factors and diabetes in sub-Saharan Africa. Prog Cardiovasc Dis 2013;56:240-50.

4. Danaei G, Singh GM, Paciorek CJ, et al. The global cardiovascular risk transition: associations of four metabolic risk factors with national income, urbanization, and Western diet in 1980 and 2008. Circulation 2013;127:1493-502, 502e1-8.

5. Mocumbi AO, Sliwa K. Women's cardiovascular health in Africa. Heart 2012;98:450-5.

6. Thienemann F, Sliwa K, Rockstroh JK. HIV and the heart: the impact of antiretroviral therapy: a global perspective. Eur Heart $J$ 2013;34:3538-46.

7. Sliwa K, Carrington MJ, Becker A, et al. Contribution of the human immunodeficiency virus/acquired immunodeficiency syndrome epidemic to de novo presentations of heart disease in the Heart of Soweto Study cohort. Eur Heart J 2012;33:866-74.

8. Stewart S, Carrington M, Pretorius S, et al. Standing at the crossroads between new and historically prevalent heart disease: effects of migration and socio-economic factors in the Heart of Soweto cohort study. Eur Heart J 2011;32:492-9.

9. Chapman MJ, Ginsberg HN, Amarenco P, et al. Triglyceride-rich lipoproteins and high-density lipoprotein cholesterol in patients at high risk of cardiovascular disease: evidence and guidance for management. Eur Heart J 2011;32:1345-61.

10. Libby P, Ridker PM, Hansson GK. Progress and challenges in translating the biology of atherosclerosis. Nature 2011;473:317-25.

11. Voight BF, Peloso GM, Orho-Melander M, et al. Plasma HDL cholesterol and risk of myocardial infarction: a mendelian randomisation study. Lancet 2012;380:572-80.

12. Huxley RR, Barzi F, Lam TH, et al. Isolated low levels of high-density lipoprotein cholesterol are associated with an increased risk of coronary heart disease: an individual participant data meta-analysis of 23 studies in the Asia-Pacific region. Circulation 2011;124:2056-64.

13. Goldbourt U, Yaari S, Medalie JH. Isolated low HDL cholesterol as a risk factor for coronary heart disease mortality. A 21-year follow-up of 8000 men. Arterioscler Thromb Vasc Biol 1997;17:107-13.

14. deGoma EM, Leeper NJ, Heidenreich PA. Clinical significance of high-density lipoprotein cholesterol in patients with low low-density lipoprotein cholesterol. J Am Coll Cardiol 2008;51:49-55.

15. Khovidhunkit W, Kim MS, Memon RA, et al. Effects of infection and inflammation on lipid and lipoprotein metabolism: mechanisms and consequences to the host. J Lipid Res 2004;45:1169-96.

16. Sliwa K, Lyons JG, Carrington MJ, et al. Different lipid profiles according to ethnicity in the Heart of Soweto study cohort of de novo presentations of heart disease. Cardiovasc J Afr 2012;23:389-95. 
17. Sliwa K, Wilkinson D, Hansen C, et al. Spectrum of heart disease and risk factors in a black urban population in South Africa (the Heart of Soweto Study): a cohort study. Lancet 2008;371:915-22.

18. Friedewald WT, Levy RI, Fredrickson DS. Estimation of the concentration of low-density lipoprotein cholesterol in plasma, without use of the preparative ultracentrifuge. Clin Chem 1972;18:499-502.

19. Klug E. South African dyslipidaemia guideline consensus statement. $S$ Afr Med J 2012;102(3 Pt 2):178-87.

20. Ridker PM, Cook N. Clinical usefulness of very high and very low levels of C-reactive protein across the full range of Framingham Risk Scores. Circulation 2004;109:1955-9.

21. Dhingra R, Gona $\mathrm{P}, \mathrm{Nam} \mathrm{BH}$, et al. C-reactive protein, inflammatory conditions, and cardiovascular disease risk. Am J Med 2007;120:1054-62.

22. Zoratti R. A review on ethnic differences in plasma triglycerides and high-density-lipoprotein cholesterol: is the lipid pattern the key factor for the low coronary heart disease rate in people of African origin? Eur J Epidemiol 1998;14:9-21.

23. Ulasi II, ljoma CK, Onodugo OD. A community-based study of hypertension and cardio-metabolic syndrome in semi-urban and rural communities in Nigeria. BMC Health Serv Res 2010;10:71.

24. Sumner AE, Zhou J, Doumatey A, et al. Low HDL-cholesterol with normal triglyceride levels is the most common lipid pattern in West Africans and African Americans with metabolic syndrome: implications for cardiovascular disease prevention. CVD Prev Contr 2010;5:75-80.

25. Farzadfar F, Finucane MM, Danaei G, et al. National, regional, and global trends in serum total cholesterol since 1980: systematic analysis of health examination surveys and epidemiological studies with 321 country-years and 3.0 million participants. Lancet 2011;377:578-86.

26. Steyn K, Sliwa K, Hawken S, et al. Risk factors associated with myocardial infarction in Africa: the INTERHEART Africa study. Circulation 2005;112:3554-61.
27. Tollman SM, Kahn K, Sartorius B, et al. Implications of mortality transition for primary health care in rural South Africa: a population-based surveillance study. Lancet 2008;372:893-901.

28. Adewole OO, Eze S, Betiku Y, et al. Lipid profile in HIV/AIDS patients in Nigeria. Afr Health Sci 2010;10:144-9.

29. Anastos K, Ndamage F, Lu D, et al. Lipoprotein levels and cardiovascular risk in HIV-infected and uninfected Rwandan women. AIDS Res Ther 2010;7:34.

30. Armstrong C, Liu E, Okuma J, et al. Dyslipidemia in an HIV-positive antiretroviral treatment-naive population in Dar es Salaam, Tanzania. J Acquir Immune Defic Syndr 2011;57:141-5.

31. Buchacz K, Weidle PJ, Moore D, et al. Changes in lipid profile over 24 months among adults on first-line highly active antiretroviral therapy in the home-based AIDS care program in rural Uganda. J Acquir Immune Defic Syndr 2008;47:304-11.

32. Ntyintyane L, Panz V, Raal FJ, et al. Leptin, adiponectin, and high-sensitivity C-reactive protein in relation to the metabolic syndrome in urban South African blacks with and without coronary artery disease. Metab Syndr Relat Disord 2009;7:243-8.

33. Nordestgaard BG. Does elevated C-reactive protein cause human atherothrombosis? Novel insights from genetics, intervention trials, and elsewhere. Curr Opin Lipidol 2009;20:393-401.

34. Albert MA, Glynn RJ, Buring J, et al. C-reactive protein levels among women of various ethnic groups living in the United States (from the Women's Health Study). Am J Cardiol 2004;93:1238-42.

35. Yusuf S, Islam S, Chow CK, et al.; Prospective Urban Rura Epidemiology (PURE) Study Investigators. Use of secondary prevention drugs for cardiovascular disease in the community in high-income, middle-income, and low-income countries (the PURE Study): a prospective epidemiological survey. Lancet 2011;378:1231-43.

36. Tleyjeh IM, Kashour T, Hakim FA, et al. Statins for the prevention and treatment of infections: a systematic review and meta-analysis. Arch Intern Med 2009;169:1658-67. 